\title{
Assistência do enfermeiro na educação em saúde, no câncer de colo do útero
}

\author{
Nurse assistance in health education in cervical cancer \\ Asistencia de enfermería en educación sanitaria en cáncer cervicouterino
}

Recebido: 09/03/2021 | Revisado: 17/03/2021 | Aceito: 21/03/2021 | Publicado: 28/03/2021

\author{
Rafaela Lima de Oliveira \\ ORCID: https://orcid.org/0000-0002-0994-3246 \\ Instituto Brasileiro de Medicina e Reabilitação, Brasil \\ E-mail: rafaela.limaoliveira@yahoo.com.br \\ Lorena Albuquerque de Sousa Lima \\ ORCID: https://orcid.org/0000-0001-5827-9196 \\ Instituto Brasileiro de Medicina e Reabilitação, Brasil \\ E-mail: lorenaalbuquerqd@gmail.com \\ Luciano Godinho Almuinha Ramos \\ ORCID: https://orcid.org/0000-0001-9958-3151 \\ Instituto Brasileiro de Medicina e Reabilitação, Brasil \\ E-mail: lucianogodinho@yahoo.com.br
}

\begin{abstract}
Resumo
Este estudo versa sobre a assistência do enfermeiro na educação em saúde na rede básica, voltada para a mulher com câncer de colo de útero. Objetiva-se destacar a assistência do enfermeiro na educação em saúde, no atendimento ao câncer de colo do útero voltada para atenção básica. Para isso, foi utilizada a seguinte questão norteadora: Como se dá a assistência do enfermeiro na educação em saúde, no câncer de colo do útero voltada para a atenção básica? Como critérios de inclusão foram utilizados artigos científicos entre os anos de 2010 a 2020, nas bases de dados, LILACS e SCIELO, em inglês, francês e espanhol. Como critérios de exclusão foram excluídos artigos fora do espaço temporal de pesquisa e que não abordavam a temática. Os resultados obtidos através dos 17 artigos científicos selecionados, trouxeram efetivação a elaboração de três categorias temáticas trazendo como principal foco a assistência do enfermeiro no atendimento a paciente com câncer do colo do útero. Conclui-se a atuação do enfermeiro nas equipes da Estratégia da Saúde da Família é de importância fundamental, suas atividades são desenvolvidas em múltiplas dimensões, como a realização das consultas de enfermagem, do exame de Papanicolau e ações educativas diversas junto à equipe de saúde e comunidade.
\end{abstract}

Palavras-chave: Educação em saúde; Câncer de colo do útero; Atenção primária; Assistência de enfermagem.

\begin{abstract}
This study deals with the assistance of nurses in health education in the basic network, aimed at women with cervical cancer. The objective is to highlight the nurse's assistance in health education, in the care of cervical cancer focused on primary care. for this, the following guiding question was used: how is the nurse's assistance in health education, in cervical cancer aimed at primary care? As inclusion criteria, scientific articles were used between the years 2010 to 2020, in the databases, LILACS and SCIELO, in English, French and Spanish. As exclusion criteria, articles were excluded outside the research time frame and that did not address the theme. The results obtained through the 17 selected scientific articles, brought about the elaboration of three thematic categories, bringing as a main focus the nurse's assistance in cervical cancer. It concludes the role of nurses in the Family Health Strategy teams of fundamental importance, their activities are developed in multiple dimensions, such as the performance of nursing consultations, the Pap test and various educational actions with the health and community team.
\end{abstract}

Keywords: Health education; Cervical cancer; Primary attention; Nursing assistance.

\section{Resumen}

Este estudio trata sobre la asistencia de enfermeras en educación para la salud en la red básica, dirigido a mujeres con cáncer de cuello uterino. El objetivo es destacar la asistencia del enfermero en la educación para la salud, en la atención del cáncer de cuello uterino con foco en la atención primaria. para ello se utilizó la siguiente pregunta orientadora: ¿cómo está orientada la asistencia del enfermero en educación para la salud, en cáncer de cuello uterino a la atención primaria? Como criterios de inclusión, se utilizaron artículos científicos entre los años 2010 a 2020, en las bases de datos LILACS y SCIELO, en inglés, francés y español. Como criterio de exclusión, se excluyeron artículos fuera del marco temporal de la investigación y que no abordaran el tema. Los resultados obtenidos a través de los 17 artículos científicos seleccionados, propiciaron la elaboración de tres categorías temáticas, teniendo como foco principal la asistencia de enfermería en cáncer cervicouterino. Se concluye el rol de las enfermeras en los equipos de Estrategia Salud de la Familia de fundamental importancia, sus actividades se desarrollan en múltiples dimensiones, como la 
realización de consultas de enfermería, el Papanicolaou y diversas acciones educativas con el equipo de salud y comunidad.

Palabras clave: Educación para la salud; Cáncer de cuello uterino; Atención primaria; Asistencia de enfermería.

\section{Introdução}

O câncer é uma doença que se caracteriza pela perda do controle da divisão celular com capacidade de invadir outras estruturas orgânicas. O câncer de colo do útero é um dos mais de 100 tipos desta doença, também chamado de câncer cervical. Este demora anos para se desenvolver, e está associado a infecção por subtipos oncogênicos do vírus HPV (papilomavírus humano), em especial os subtipos HPV-16 e o HPV-18. Por ser uma infecção muito comum, cerca de $80 \%$ das mulheres ativas sexualmente, irão adquirir ao longo de suas vidas alguns dos subtipos deste vírus. Nos dias atuais cerca de 290 milhões de mulheres são portadoras do papilomavírus humano (HPV), e dentre essas mulheres, cerca de 32\% delas irão desenvolver o câncer do colo do útero, o que é equivalente a uma incidência de 500 mil casos de câncer do colo do útero por ano. Segundo o Instituto Nacional do Câncer, o câncer de colo de útero é o terceiro tumor maligno mais frequente na população feminina, sendo a quarta causa de morte em mulheres no Brasil. (Instituto Nacional de Câncer José de Alencar Gomes da Silva 2020).

Os fatores de risco para desenvolvimento deste tipo de câncer se dão através da multiplicidade de parceiros sexuais, tabagismo, atividade sexual precoce, uso prolongado de pílulas anticoncepcionais e pré disposição genética. (Brasil, 2011).

De acordo com Brasil 2014, a infecção pelo vírus do HPV, pode ser parcialmente evitada com o uso de preservativos, assim como o câncer de colo do útero pode ser prevenido através do exame preventivo Papanicolau ou colpocitologia cervical. (Brasil, 2017).

O exame preventivo Papanicolau é um método para identificação e prevenção da infecção do HPV e do desenvolvimento do câncer do colo do útero, onde é realizado de forma que se identifica alteração celular, antes mesmo que a infecção apresente sintomas, como verrugas genitais ou feridas no colo do útero e sangramentos repentinos acompanhados de dor. (Brasil, 2015).

Para a abordagem a essas mulheres no atendimento em rede básica de saúde, a realização do exame preventivo tornase uma maneira eficaz de rastreamento da doença. A realização deste procedimento, de forma indolor, rápida e simples, é periódica a cada três anos após dois exames anuais consecutivos negativos, sua indicação é para mulheres do grupo alvo de 25 a 64 anos de idade que tenham iniciado a vida sexual, podendo ser realizado em postos de saúde unidades de saúde de rede pública e em clínicas que tenham profissionais capacitados.

Considerando a resolução COFEN N ${ }^{\circ} 381 / 2011$, que entende a coleta de material para colpocitologia oncótica pelo método Papanicolau como um procedimento complexo que demanda competência técnica e cientifica em sua execução, Art. $\mathbf{1}^{\circ}$, vemos o enfermeiro como o profissional capacitado para a abordagem a essa mulher, em seu primeiro contato com a unidade básica, que procura atendimento com grandes medos e receios de complicações desta doença. De acordo com o código de ética dos profissionais de enfermagem, a coleta de material para colpocitologia oncótica pelo método Papanicolau é privativa do enfermeiro, observadas as disposições legais da profissão. (Conselho Federal de Enfermagem, 2011)

A atitude preventiva e a educação em saúde para o cuidado e melhoria da qualidade de vida das mulheres são um importante componente para a detecção precoce da infecção pelo papilomavirus humano e também para a detecção do câncer de colo do útero. $\mathrm{Na}$ atenção primaria existe toda uma sistematização para rastreamento e deteç̧ão do câncer de colo de útero ressaltando os benefícios da realização do Papanicolau, juntamente com a educação dos profissionais e da população. (Ramos de Souza, K et al. 2015).

A educação em saúde é um dos pontos primordiais para a prevenção deste tipo de câncer, é uma estratégia para alcançar resultados positivos e efetivos, os estudos de Santos MSD et al. (2011) descrevem ações da enfermagem, como, informações ao estimulo ao sexo seguro, ao não uso do tabaco, mudança comportamental referente a vida sexual, prática de exercícios físicos e 
alimentação rica em nutrientes, juntamente com o acompanhamento ginecológico regular são formas de prevenir e conscientizar sobre o risco de infecção pelo HPV e desenvolvimento do câncer de colo do útero. (Santiago TR, et al. 2014).

Diante disto, esta pesquisa tem o intuito de demonstrar como a assistência do enfermeiro, na atenção primária em saúde, é de extrema importância para a prevenção e acompanhamento das mulheres que sofrem deste agravo.

\section{Metodologia}

Segundo Mendes KDS, Silveira RCCP, Galvão CM, 2008, A prática baseada em evidências é uma abordagem que encoraja o desenvolvimento e/ ou utilização de resultados de pesquisas na prática clínica, devido à quantidade e complexidade de informações na área da saúde, há necessidade de produção de métodos de revisão de literatura e para alcançar o objetivo proposto, foi utilizado este tipo de metodologia.

Para o levantamento dos artigos foi realizada uma busca online na BVS, LILACS, SCIELO. Todo o processo de desenvolvimento da pesquisa e seleção dos artigos foi conduzido por dois pesquisadores independentes. No processo de montagem, foi utilizado além da busca na literatura dos estudos primários, extração de dados e avaliação dos estudos primários incluídos.

Elegeu-se a seguinte questão norteadora: Como se dá a assistência do enfermeiro na educação em saúde no câncer de colo do útero voltada para a atenção básica?

A partir da busca dos estudos selecionados conforme os critérios de inclusão, foi utilizado artigos científicos entre os anos de 2010 a 2020, em inglês, francês e espanhol, artigos disponíveis na íntegra, artigos originais que abrangessem os objetivos propostos e abordassem o tema: assistência do enfermeiro na educação em saúde, no câncer de colo do útero, totalizando 30 artigos.

Conforme os critérios de exclusão, os artigos que não apresentavam a temática, artigos de revisão, artigos que não se relacionavam aos objetivos desta pesquisa, dissertações ou teses, foram descartados. Dessa forma foram excluídos um total de 13 artigos. Sendo que, após a leitura, selecionou-se apenas 17 artigos para a elaboração final do estudo pelos bancos de dados.

A seguir, um fluxograma sobre os artigos que compuseram a amostra final da revisão. 
Fluxograma 1. Busca e seleção dos 17 artigos. Rio de janeiro, RJ, Brasil, 2020.

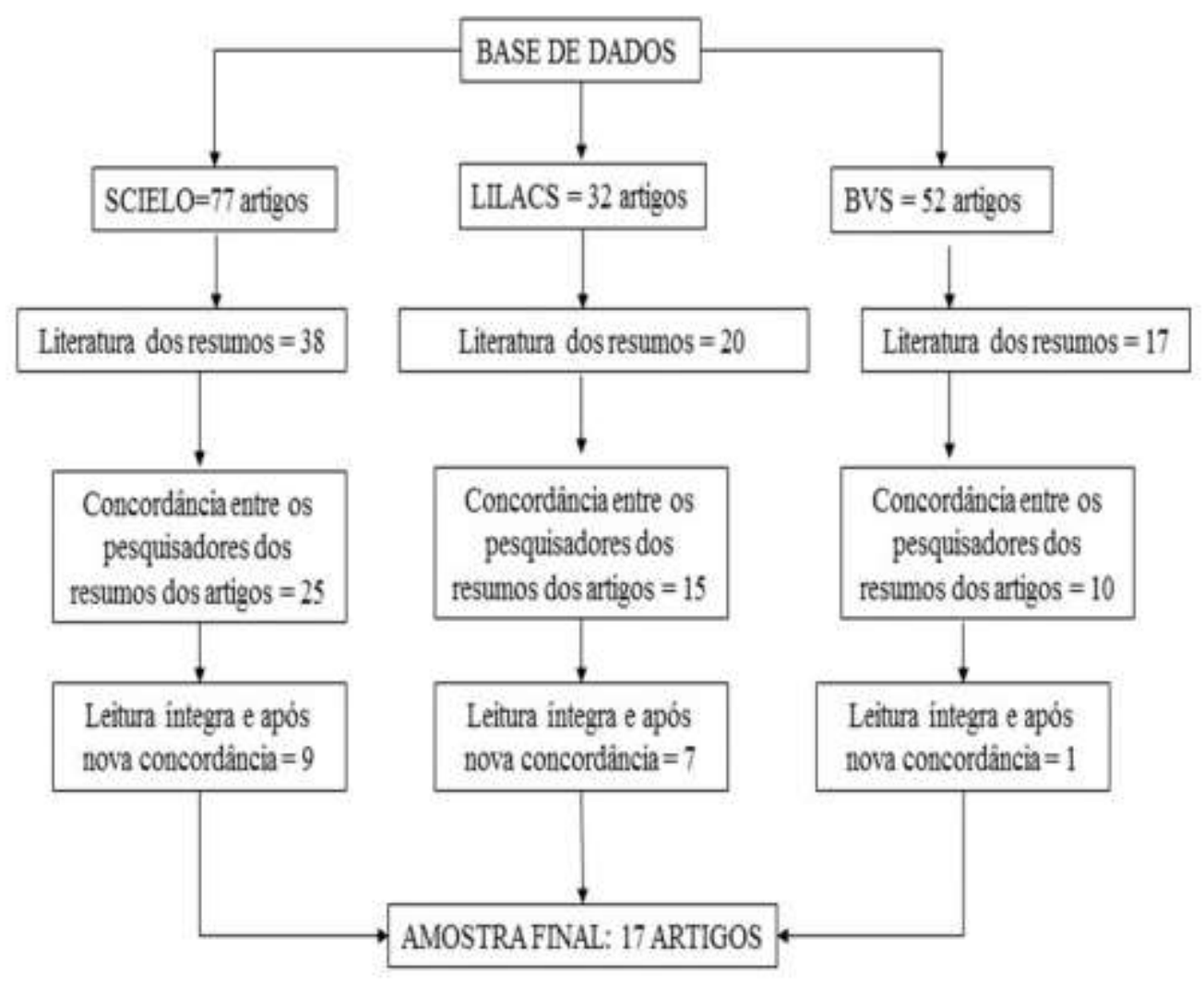

Fonte: Dados da pesquisa.

Observando o Fluxograma 1, podemos analisar a ordem de busca e a predileção dos artigos que compuseram a elaboração da revisão, sendo iniciado pelo banco de dados e sequencialmente a seleção dos artigos, exclusão e a escolha para amostra final.

\section{Resultados e Discussão}

Quadro 1. Quadro expositivo dos artigos pesquisados neste estudo. Rio de Janeiro, RJ, Brasil, 2020.

\begin{tabular}{|l|l|l|}
\hline AUTOR/ANO & TÍTULO & OBJETIVOS \\
\hline CARVALHO, PG et al/ & $\begin{array}{l}\text { Trajetórias assistenciais de mulheres } \\
\text { entre diagnóstico e início de } \\
\text { tratamento do câncerde colo uterino. }\end{array}$ & $\begin{array}{l}\text { Analisar as trajetórias na assistência das mulheres } \\
\text { residentes no Município do Rio de Janeiro diagnosticadas } \\
\text { com câncer de colo uterino que foram encaminhadas para } \\
\text { tratamento em unidade de referência na atenção oncológica. }\end{array}$ \\
\hline $\begin{array}{l}\text { OLIVEIRA, JLT et al// } \\
2017\end{array}$ & $\begin{array}{l}\text { Intervenções de enfermagem na } \\
\text { prevenção do câncer cérvico- } \\
\text { uterino: perspectivas das clientes. }\end{array}$ & $\begin{array}{l}\text { Analisar as intervenções de enfermeiros que podem } \\
\text { proporcionar mudanças de comportamentos, hábitos e } \\
\text { estilos de vida para prevenção do câncer cérvico-uterino, na } \\
\text { perspectiva das clientes. }\end{array}$ \\
\hline
\end{tabular}




\begin{tabular}{|c|c|c|}
\hline $\begin{array}{l}\text { CARVALHO, BG et al/ } \\
2015\end{array}$ & $\begin{array}{l}\text { Integralidade do cuidado no } \\
\text { Programa de Controle do Câncer } \\
\text { de Colo Uterino: visão das } \\
\text { usuárias com alteração na } \\
\text { citologia oncótica. }\end{array}$ & $\begin{array}{l}\text { Avaliar a integralidade no cuidado de mulheres atendidas } \\
\text { pelo Programa de Controle do Câncer de Colo Uterino. }\end{array}$ \\
\hline SOUZA, R et al/ 2015 & $\begin{array}{lcr}\text { Educação } & \text { popular } & \text { como } \\
\text { instrumento } & \text { participativo } & \text { para } \\
\text { prevenção } & \text { do } & \text { câncer } \\
\text { ginecológico: } & \text { percepção } & \text { de } \\
\text { mulheres. } & & \\
\end{array}$ & $\begin{array}{l}\text { Avaliar a percepção de mulheres sobre o câncer do colo do } \\
\text { útero, através da prática de educação popular como } \\
\text { instrumento participativo. }\end{array}$ \\
\hline $\begin{array}{l}\text { CORREIO, KDL et al/ } \\
2015\end{array}$ & $\begin{array}{l}\text { Controle do câncer do colo do } \\
\text { útero: ações desenvolvidas pelo } \\
\text { enfermeiro à luz do discurso do } \\
\text { sujeito coletivo }\end{array}$ & $\begin{array}{l}\text { Compreender o processo do trabalho do enfermeiro da } \\
\text { Estratégia de Saúde da Família (ESF) no controle do câncer } \\
\text { do colo do útero do munícipio de Carpina-PE }\end{array}$ \\
\hline $\begin{array}{l}\text { SANTIAGO, TR et al/ } \\
2014\end{array}$ & $\begin{array}{l}\text { Conhecimento e prática das } \\
\text { mulheres atendidas na unidade de } \\
\text { saúde da família sobre o } \\
\text { Papanicolaou. }\end{array}$ & $\begin{array}{l}\text { O objetivo do estudo foi descrever o conhecimento e a } \\
\text { prática sobre o Papanicolaou das mulheres entre } 25 \text { a } 59 \\
\text { anos atendidas pela Estratégia de Saúde da Família. }\end{array}$ \\
\hline SILVA, MM et al/ 2013 & $\begin{array}{l}\text { Atenção básica em saúde: } \\
\text { prevenção do câncer de colo do } \\
\text { útero na consulta de enfermagem. }\end{array}$ & $\begin{array}{l}\text { Objetivou-se analisar os eixos teórico-conceituais } \\
\text { estruturantes da consulta de enfermagem ginecológica na } \\
\text { atenção básica }(\mathrm{AB}) \text { e discutir as principais condutas } \\
\text { implementadas para prevenção do câncer do colo do útero } \\
\text { (CCU). }\end{array}$ \\
\hline VIANA, MRP et al/ 2013 & $\begin{array}{l}\text { Formação do enfermeiro para } \\
\text { a prevenção do câncer de colo } \\
\text { uterino. }\end{array}$ & $\begin{array}{l}\text { Objetivou-se analisar a formação do enfermeiro para a } \\
\text { prevenção do câncer de colo uterino no contexto da } \\
\text { estratégia saúde da família. }\end{array}$ \\
\hline $\begin{array}{l}\text { NASCIMENTO, LC et al/ } \\
2012\end{array}$ & $\begin{array}{l}\text { Conhecimento cotidiano de } \\
\text { mulheres sobre a prevenção do } \\
\text { câncer de colo do útero. }\end{array}$ & $\begin{array}{l}\text { O objetivo deste estudo foi apreender as representações } \\
\text { sociais elaboradas por } 64 \text { mulheres da cidade de Teresina } \\
\text { durante os meses de outubro e novembro de } 2009 \text {, acerca da } \\
\text { prevenção do câncer de colo do útero, bem como analisar } \\
\text { como essas representações influem na realização do exame } \\
\text { de prevenção. }\end{array}$ \\
\hline DANTAS, CN et al/ 2012 & $\begin{array}{l}\text { A consulta de enfermagem na } \\
\text { prevenção do câncer cérvico- } \\
\text { uterino para mulheres que a } \\
\text { vivenciaram. }\end{array}$ & $\begin{array}{l}\text { Identificar o significado da consulta de enfermagem na } \\
\text { prevenção do câncer cérvico-uterino realizada segundo os } \\
\text { preceitos da enfermagem humanística, para as mulheres que } \\
\text { a vivenciaram. }\end{array}$ \\
\hline
\end{tabular}




\begin{tabular}{|c|c|c|}
\hline TOMASI, E et al/ 2012 & $\begin{array}{l}\text { Estrutura e processo de trabalho } \\
\text { na prevenção do câncer de colo de } \\
\text { útero na Atenção Básica à Saúde } \\
\text { no Brasil: Programa de Melhoria } \\
\text { do Acesso e da Qualidade - } \\
P M A Q \text {. }\end{array}$ & $\begin{array}{l}\text { Descrever e analisar a adequação da estrutura e processo das } \\
\text { equipes do Programa de Melhoria do Acesso e da Qualidade } \\
\text { (PMAQ-AB) na prevenção do câncer de colo de útero na } \\
\text { Atenção Básica no Brasil. }\end{array}$ \\
\hline MELO, MCSC et al/ 2012 & $\begin{array}{l}\text { O enfermeiro na prevenção do } \\
\text { câncer do colo do útero: } o \\
\text { cotidiano da atenção primária. }\end{array}$ & $\begin{array}{l}\text { Analisar o desenvolvimento da prevenção e detecção } \\
\text { precoce do câncer do colo do útero no cotidiano assistencial } \\
\text { da enfermeira que atua nas equipes da Estratégia Saúde da } \\
\text { Família, a partir de suas atribuições, propostas pelo } \\
\text { Ministério da Saúde. }\end{array}$ \\
\hline $\begin{array}{l}\text { VASCONCELOS, CTM et } \\
\text { al/ } 2011\end{array}$ & $\begin{array}{l}\text { Conhecimento, atitude e prática } \\
\text { relacionada ao exame } \\
\text { colpocitológico entre usuárias de } \\
\text { uma unidade básica de saúde. }\end{array}$ & $\begin{array}{l}\text { Avaliar o conhecimento, a atitude e a prática do exame } \\
\text { colpocitológico entre usuárias de uma unidade básica de } \\
\text { saúde (UBS) e verificar sua associação com variáveis } \\
\text { sociodemográficas. }\end{array}$ \\
\hline SANTOS, MSS et al/ 2011 & $\begin{array}{l}\text { Saberes e práticas de mulheres } \\
\text { idosas na prevenção do câncer } \\
\text { cérvico-uterino. }\end{array}$ & $\begin{array}{l}\text { Descrever os saberes e práticas de idosas sobre o câncer } \\
\text { cérvico-uterino e analisar as ações preventivas para esta } \\
\text { neoplasia }\end{array}$ \\
\hline SOARES, MC Et al/ 2011 & $\begin{array}{l}\text { Câncer de colo uterino: Atenção } \\
\text { integral à mulher nos serviços de } \\
\text { saúde }\end{array}$ & $\begin{array}{l}\text { Compreender como os serviços de saúde do Sistema Único } \\
\text { de Saúde estão organizados para contemplar a integralidade } \\
\text { na atenção à mulher com câncer de colo uterino. }\end{array}$ \\
\hline VALE, DBAP et al/ 2010 & $\begin{array}{l}\text { Avaliação do rastreamento do } \\
\text { câncer do colo do útero na } \\
\text { Estratégia Saúde da Família no } \\
\text { Município de Amparo, São Paulo, } \\
\text { Brasil. }\end{array}$ & $\begin{array}{l}\text { Analisar se o rastreamento do câncer do colo uterino em } \\
\text { Amparo, Estado de São Paulo, Brasil, avançou no sentido } \\
\text { de seguir as recomendações vigentes ao longo de sete anos } \\
\text { da ESF. }\end{array}$ \\
\hline CIRINO, FMSB et al/ 2010 & $\begin{array}{l}\text { Conhecimento, atitude e práticas } \\
\text { na prevenção do câncer de colo } \\
\text { uterino e HPV em adolescentes. }\end{array}$ & $\begin{array}{l}\text { Identificar o conhecimento, atitude e prática na prevenção } \\
\text { do câncer de colo uterino e infecção pelo HPV na população } \\
\text { adolescente e avaliar as situações que as tornam } \\
\text { vulneráveis. }\end{array}$ \\
\hline
\end{tabular}

Fonte: Dados da pesquisa.

Analisando o Quadro 1 podemos observar a estrutura e organização dos artigos selecionados para a elaboração e desenvolvimento desta revisão integrativa, tendo em sua composição, autores, título do artigo e o assunto abordado.

A busca dos artigos científicos foi realizada entre setembro e dezembro de 2020, e para a apresentação das etapas de seleção dos artigos de acordo com as informações adquiridas, foi elaborado uma tabela como metodologia com os principais dados dos artigos científicos escolhidos.

Dos dezessete artigos selecionados no campo deste estudo, todos tiveram publicação entre 2010 e 2018 , sendo em maior quantidade as apresentações dos artigos de 2013 e 2015. 
Os resultados obtidos através dos 17 artigos científicos selecionados, trouxeram efetivação a elaboração de três categorias temáticas: Tipos de intervenções que o enfermeiro realiza na prevenção do Câncer do Colo do Útero, O papel do enfermeiro no atendimento à mulher com Câncer do Colo do Útero e $\mathrm{O}$ acolhimento à mulher nas consultas das unidades básicas de saúde.

\section{Categoria 1 - Tipos de intervenções que o enfermeiro realiza na prevenção do Câncer do Colo do Útero.}

Ao analisar os artigos ficou evidente que as ações desenvolvidas pelo profissional enfermeiro são fundamentais na prevenção e detecção precoce do câncer do colo uterino, sendo o mesmo atuante na atenção primária onde realiza relevantes condutas visando à promoção e prevenção de agravos na saúde da mulher.

De acordo com Vasconcelos et al, 2011, as atribuições que a enfermagem executa utilizando os lugares da comunidade para a realização de promoção, orientação, atendimentos individuais ou coletivos, por meio do processo de enfermagem visando à melhoria da qualidade de vida, torna-se um fator importante na prevenção do câncer do colo do útero dentro da atenção primária. (Vale, et al. 2010).

Durante a consulta de enfermagem pode-se realizar atividades educativas com cada mulher, orientando e enfatizando aspectos referentes à prevenção e realçando medidas preventivas.

Podemos citar três tipos de intervenções que podem ser executadas individualmente ou coletivamente pela enfermagem para a prevenção ou controle do câncer de colo do útero, são elas:

Comportamentais: As que estimulam as usuárias a realizarem o exame de detecção precoce; Cognitivas: As que conscientizam e informam essas mulheres sobre as ações que devem ser realizadas para a prevenção e controle das patologias; e Sociais: Onde a enfermagem atua de forma indireta, onde as próprias pessoas ou a unidade possam ser treinadas para essa ação e também por meio da atuação direta do enfermeiro, possibilitando o aumento do conhecimento sobre as medidas preventivas.

Para atuação satisfatória, faz-se necessário que o profissional conheça a cultura e a realidade da população alvo, pois o comportamento preventivo está intimamente ligado também aos fatores sociais, psicológicos e ambientais.

A estratégia mais eficaz no combate ao câncer do colo do útero é a prevenção mediante diagnóstico em estágios iniciais, porém, o rastreamento precoce entre as mulheres nem sempre é possível em virtude da dificuldade de acesso dessa população aos serviços de saúde. Deste modo, reduzem-se as chances de prevenção e intensificam-se os riscos da doença. (Guimarães, Aquino, Pinheiro, Moura. 2012).

Entre as estratégias desenvolvidas nas Unidades básicas de saúde na perspectiva de um melhor atendimento à saúde da mulher, evidencia-se o método de colpocitologia oncótica ou exame de Papanicolau que objetiva detectar preferencialmente as lesões precursoras do câncer do colo do útero ou este o mais precocemente possível. (Carvalho, et al. 2018).

Nessa metodologia, considerada de realização simples, com rigor técnico, por meio da coleta de um esfregaço, são obtidas amostras celulares do epitélio da ectocérvice e endocérvice.

Uma das formas de abordagem que podem ser satisfatórias com as mulheres é o acolhimento, e quando é realizado de forma correta cria-se um vínculo de credibilidade e confiança entre profissional e a paciente facilitando assim de forma positiva e humanizada a passagem de informações. (Silva, et al. 2012).

Para um melhor resultado das ações do enfermeiro nas unidades básicas de saúde, destacam-se a importância de um sistema de controle das mulheres da sua área de abrangência por meio de um rastreamento. O sucesso desta ação está diretamente ligado a um número suficiente de profissionais capacitados para realizar os exames, bem como o diagnóstico correto e o tratamento das mulheres e como indicado nas diretrizes, contar sempre com um sistema de referência e contrarreferência efetivo nos diferentes níveis de atenção à saúde. (Oliveira, et al. 2017). 
O enfermeiro atuante na Estratégia Saúde da Família desenvolve ações e intervenções que preconizam na Política Nacional da Atenção Básica a prevenção de doenças ou agravos à saúde da população, que firmam a comunicação com os usuários através do acolhimento e vínculo, como explicado na PNAB. (Souza, Mandu, Elias 2013).

Pode-se afirmar que o profissional mais dinâmico no rastreamento do câncer do colo do útero é o enfermeiro, pois ele fornece informações e segurança às mulheres sobre o exame, abrindo espaços de acolhimento nas consultas de enfermagem.

\section{Categoria 2 - O papel do enfermeiro no atendimento à mulher com câncer do colo de útero.}

A importância de conhecer as práticas de atenção à saúde e a maneira como se efetivam no cotidiano do trabalho para a produção do cuidado tanto para a área da gestão quanto da organização dos serviços de saúde, são de relevância para a configuração da integralidade. (Cirino, et al. 2010).

Não somente como princípio do SUS e Brasil, 2020, mas também como boas práticas de saúde para a melhoria do cuidado, sobretudo na atenção à mulher, expressa-se também na escuta, no vínculo e nas concepções que as mulheres, os trabalhadores de saúde e os gestores do sistema de saúde apresentam das formas de fazer saúde, e como atentam para a possibilidade de coparticipação no processo de construção de saúde para todos. (Governo do Distrito Federal, 2018).

As Unidades de Atenção Primária à Saúde (UAPS) são consideradas porta de entrada do usuário no sistema de saúde, espaço em que o enfermeiro é importante integrante da equipe multiprofissional da Estratégia Saúde da Família. (Brasil, 2010).

Conforme o tamanho da área de abrangência, distribuem-se equipes que têm como desafio, o trabalho integrado e a responsabilidade pelas pessoas ali residentes. Nesse contexto, os enfermeiros exercem atividades técnicas específicas de sua competência, administrativas e educativas. Através do vínculo com as usuárias, concentra esforços para reduzir os tabus, mitos e preconceitos e buscando o convencimento da clientela feminina sobre os seus benefícios da prevenção. Para o planejamento das atividades e estratégias, são consideradas e respeitadas as peculiaridades regionais, envolvimento das lideranças comunitárias, profissionais da saúde, movimentos de mulheres e meios de comunicação. (Melo, et al. 2012).

O enfermeiro que está inserido na atenção primária tem o papel de gerente e provedor da assistência à saúde e como função primordial o rastreamento desta neoplasia por intermédio das consultas individuais e coleta do exame citopatológico. (Melo, et al. 2012).

A responsabilidade do enfermeiro é de contribuir com a compreensão da realidade em todas as suas dimensões, dispondo a romper com as ações do modelo queixas-condutas, para que a realidade seja compreendida em sua integralidade. (Dantas, et al. 2012).

De acordo com Viana MP, et al. 2013 entre as suas ações, o processo de educação em saúde serve para subsidiar a adesão satisfatória das usuárias aos serviços, facilitando o entendimento e sensibilizando-as à realização do exame preventivo, bem como, para mudanças de comportamento que viabilizem uma vida saudável e de boa qualidade. (Omasi, et al. 2015).

É parte das atribuições do enfermeiro, realizar assistência integral às mulheres que procuram a unidade de saúde, neste caso, a realização da consulta de enfermagem e coleta de material para a citologia oncótica, além disto, há ainda que manter a disponibilidade dos materiais necessários para a realização do exame colpocitológico, fato que nem sempre se faz cumprir a contento, na realidade mencionada por alguns profissionais.

\section{Categoria 3 - $O$ acolhimento à mulher nas consultas das unidades básicas de saúde.}

"Acolher é dar acolhida, admitir, aceitar, dar ouvidos, dar crédito a agasalhar, receber, atender, admitir" (Brasil, 2010). O acolhimento como ato ou efeito de acolher expressa, em suas várias definições, uma ação de aproximação, ou seja, uma atitude de inclusão. Essa atitude implica, por sua vez, estar em relação com algo ou alguém. (Nascimento, et al. 2012). De acordo com Soares, et al. 2011 é exatamente nesse sentido, de ação de inclusão, que queremos afirmar o acolhimento como uma das diretrizes 
de maior relevância ética e estética da Política Nacional de Humanização do SUS, onde a ética se refere ao compromisso com o reconhecimento do outro, na atitude de acolhê-lo em suas diferenças, suas dores, suas alegrias, seus modos de viver, sentir e estar na vida, porque traz para as relações e os encontros do dia-a-dia a invenção de estratégias que contribuem para a dignificação da vida e do viver e, assim, para a construção de nossa própria humanidade e a política porque implica o compromisso coletivo de envolver-se neste "estar com", potencializando protagonismos e vida nos diferentes encontros. (Oliveira, et al. 2017).

$\mathrm{O}$ acolhimento implica no estabelecimento de relações de aproximação de modo humanizado de acordo com Correio KL, et al. 2015, na perspectiva do desenvolvimento da autonomia e sua efetivação estará relacionada com a utilização dos recursos disponíveis que se adequem e concorram para a resolução dos problemas dos usuários. (Carvalho, et al. 2015).

O acolher inicia-se no primeiro instante de um contato entre pessoas, é a atenção, o ouvir, enfim, é uma relação de respeito mútua, necessária ao desenvolvimento do trabalho, que vai aos poucos, organizando uma sociedade menos individualista e mais passível de mudanças, de acordo com a necessidade do outro. (Medeiros, et al. 2010).

Foi realizado uma pesquisa no banco de dados da SCIELO sobre um estudo avaliativo, de natureza qualitativa, em que se realizou um monitoramento sistemático das mulheres atendidas pelo PNCCU em uma UBS no ano de 2012. Estima-se que, na faixa etária de 25 a 64 anos, público alvo do PNCCU, residiam no território dessa UBS 2.600 mulheres, a pesquisa foi submetida e aprovada pelo Comitê de Ética da Universidade Estadual de Londrina, sob o parecer $n^{\circ}$ 053/2013, CAAE $n^{\circ}$ 15588213.0.0000.5231, onde as mulheres participantes do estudo referiram fragilidades e potencialidades quanto ao acesso e ao acolhimento do serviço de saúde como medidas complementares para a garantia da continuidade do cuidado e do consequente alcance da integralidade do serviço prestado. De acordo com as falas das usuárias, a dificuldade da continuidade do cuidado já fica evidente no momento da coleta do preventivo, sendo ressaltado como algo constrangedor, chato, vergonhoso e horrível, resultando na recusa da mulher em realizar o tratamento. As consequências do primeiro encontro entre profissional e usuário alteram de forma decisiva a passagem do usuário pela unidade e a resolução de seus problemas. Mesmo expressando esses sentimentos, a maioria das participantes do estudo reconhecem o exame como necessário para o diagnóstico de patologias e que sua realização deve ser constante. Os resultados nos levam a pensar sobre o problema do acesso ao tratamento necessário, durante o processo de construção do SUS, foi propagado que a implementação da Estratégia da Saúde da Família, suas ações e serviços, ampliaram o acesso aos usuários, essa afirmação é válida quando se pensa na dimensão quantitativa do acesso e aumento do número de pessoas atendidas pelo sistema na Atenção Básica. No entanto, torna-se indispensável passar do enfoque estritamente quantitativo para o qualitativo, ou seja, considerar, além do primeiro atendimento, a continuidade do cuidado na rede de atenção e a qualidade do cuidado ao usuário.

As potencialidades e fragilidades no cuidado às mulheres que tiveram resultado do exame de citologia alterado nos remete à proposta do acolhimento como diretriz operacional dos serviços de saúde, este acontece, na relação usuário-serviço como um dispositivo para propiciar o acesso. O vínculo entre equipe-população, desencadeia o cuidado integral e modifica a clínica, processo mediado pela capacidade dos trabalhadores de recepcionar, atender, escutar, dialogar, tomar decisão, amparar, orientar e negociar.

Entretanto, apesar do que foi relatado pelas outras entrevistadas, uma usuária declarou não haver escuta por parte do trabalhador de saúde. O contato para informar sobre o diagnóstico, foi feito de maneira impessoal, e o encaminhamento para outro serviço realizado não obteve esclarecimento sobre o agravo e tratamento proposto. Esse depoimento nos leva a inferir que, nessa situação, o atendimento não esteve centrado no usuário, o que faz com que o mesmo assuma a condição de objeto, sendo colocado à margem do plano terapêutico, com a ausência de interação e vínculo pode-se gerar expectativas que não são alcançadas e frustrações com relação ao cuidado prestado. 


\section{Conclusão}

Os dezessete artigos científicos selecionados destacaram que a maioria das mulheres possuem conhecimento sobre o câncer de colo do útero, e que ao longo da vida trocaram informações com outras sobre o tema proposto como também relataram que já obtiveram acesso ao atendimento de saúde através da Atenção Primária. Como meio de prevenção primária, as ações educativas foram destacadas, e na prevenção secundária, a realização do exame preventivo Papanicolau pelos profissionais de saúde. As práticas que podem interferir na prevenção do Câncer de Colo do Útero, foram evidenciadas nas características comportamentais das mulheres em não usar preservativo nas relações sexuais e não realizar a colpocitologia oncótica de forma periódica. Foi evidenciado que o cuidado contínuo das mulheres na prevenção do câncer do colo de útero, é reflexo de um acolhimento ineficaz, o que caracteriza uma propagação maior da doença.

É evidente que o acolhimento é como uma ação que por meio de diálogos corresponsabiliza o usuário no processo da produção da saúde.

No cenário da prevenção do câncer de colo do útero, a atuação do enfermeiro nas equipes da ESF revelou-se de importância fundamental, suas atividades são desenvolvidas em múltiplas dimensões, entre elas: realização das consultas de enfermagem e do exame de Papanicolau, ações educativas diversas junto à equipe de saúde e comunidade, gerenciamento e contatos para o provimento de recursos materiais e técnicos, controle da qualidade dos exames, verificação, comunicação dos resultados e encaminhamentos para os devidos procedimentos quando necessário.

A análise realizada neste estudo trouxe claridade à importância do enfermeiro, assim como de sua integração com os outros componentes da equipe de saúde e com a comunidade. Nessa atuação de aspecto e olhar múltiplo é que se constrói o vínculo necessário à prática que se resulta benéfica e se alicerça no conhecimento da realidade local e avaliação constante dos resultados para sistematizar as ações que visam à redução do dano pela doença.

\section{Referências}

Brasil. (2010). Ministério da Saúde. Acolhimento nas práticas de produção de saúde: Série B. Textos Básicos de Saúde. https://bvsms.saude.gov.br/bvs/publicacoes/acolhimento_praticas_producao_saude.pdf

Brasil. (2011). Ministério da saúde. Instituto Nacional de Câncer (INCA). Diretrizes Brasileiras para o rastreamento Do Câncer do Colo do Útero. http://bvsms.saude.gov.br/bvs/publicacoes/inca/rastreamento_cancer_colo_utero.pdf

Brasil. (2014). Ministério da saúde. Secretaria de vigilância em saúde departamento de vigilância de doenças transmissíveis coordenação-geral do programa nacional de imunizações. Informe técnico sobre a vacina papilomavírus humano (hpv) na atenção básica. https://portalarquivos2.saude.gov.br/images/pdf/2015/junho/26/informe-t--cnico-introdu----o-vacina-hpv-18-2-2014.pdf

Brasil. (2015). Ministério da saúde. Biblioteca virtual em saúde. Papanicolau (exame preventivo de colo de útero). Set. https://bvsms.saude.gov.br/dicas-emsaude/2069-papanicolau-exame-preventivo-de-colo-de-utero

Brasil. (2017). Ministério da saúde. secretaria de vigilância em saúde departamento de vigilância de doenças transmissíveis coordenação geral do programa nacional de imunizações. Guia prático sobre hpv perguntas e respostas. https://portalarquivos2.saude.gov.br/images/pdf/2017/dezembro/07/Perguntas-erespostas-HPV-.pdf

Brasil. (2020). Ministério da saúde. Secretaria de Atenção à Saúde Departamento de Atenção Básica. Cadernos de Atenção Básica, Acolhimento a demanda espontânea. http://bvsms.saude.gov.br/bvs/publicacoes/acolhimento_demanda_espontanea_cab28v1.pdf

Campos E A. (2018). Os sentidos do Papanicolaou para um grupo de mulheres que realizou a prevenção do câncer cervical. Cad. saúde colet. 26(2). http://dx.doi.org/10.1590/1414-462x201800020287

Carvalho B G, et al. (2015). Integralidade do cuidado no Programa de Controle do Câncer de Colo Uterino: Visão das usuárias com alteração na citologia oncótica. Saúde debate. https://doi.org/10.1590/0103-1104201510600030012.

Carvalho, P. G., et al. (2018). Trajetórias assistenciais de mulheres entre diagnóstico e início de tratamento do câncer de colo uterino. Artigo original. https://www.scielosp.org/article/sdeb/2018.v42n118/687-701/.

Castaneda, L., et al. (2019). Prevalência de incapacidades e aspectos associados em mulheres com câncer de colo do útero, Rio de Janeiro, Brasil. Cad. saúde colet. 27(3). http://dx.doi.org/10.1590/1414-462x201900030440

Cirino, F. B., et al. (2010). Conhecimento, atitude e práticas na prevenção do câncer de colo uterino e HPV em adolescentes. Esc. Anna Nery. 14(1). https://www.scielo.br/scielo.php?script=sci_arttext\&pid=S1414-81452010000100019 
Conselho Federal de Enfermagem. (2011) RESOLUÇÃO COFEN N 381/2011. http://www.cofen.gov.br/resoluo-cofen-n-3812011_7447.html

Correio, K. L, et al. (2015). Controle do câncer do colo do útero: ações desenvolvidas pelo enfermeiro à luz do discurso do sujeito coletivo. Rev. Pesqui. https://pesquisa.bvsalud.org/portal/resource/pt/bde-26833

Costa, T. M. L., et al. (2019). Papilomavírus humano e fatores de risco para adenocarcinoma cervical no estado de Pernambuco, Brasil. Rev. Bras. Saude Mater. Infant. 19(3). https://doi.org/10.1590/1806-93042019000300009

Dantas, C. N., et al. (2012). A consulta de enfermagem na prevenção do câncer cérvico-uterino para mulheres que a vivenciaram. Rev Rene. http://www.periodicos.ufc.br/rene/article/view/3985/3144

Governo do Distrito Federal. (2018). Secretaria de estado de saúde subsecretaria de atenção integral à saúde. Protocolo de Atenção à Saúde guia de enfermagem da atenção primária à saúde: http://www.saude.df.gov.br/wp-conteudo/uploads/2018/04/enfermagem-1-protocolo_final_parte_1.pdf

Guimarães, J. A. F., Aquino, P. S., Pinheiro, A. K. B., Moura, J. G. (2012). Pesquisa brasileira sobre prevenção do câncer de colo uterino. http://www.revistareneu fc.br/revista/index.php/revista/article/view/34.

Instituto Nacional de Câncer José de Alencar Gomes da Silva (2020). Câncer. O que é câncer? https://www.inca.gov.br/oqueecancer\#: :text=C\%C3\%A2ncer\% 20\%C3\%A9\%20um\%20termo\%20que,adjacentes\%20ou\%20\%C3\%B3rg\%C3\%A3os\%20a\%20dist\%C3\%A2ncia. Instituto Nacional de Câncer José de Alencar Gomes da Silva (2020). Tipos de câncer: Câncer do colo do útero - versão para Profissionais de Saúde. Nov27. https://www.inca.gov.br/tipos-de-cancer/cancer-do-colo-do-utero/profissional-de-saude

Marta Soraia, L., Menêses, M. B. P., Toralles, C. M. C. M. (2019). Evolução da técnica de PCR: sua contribuição no diagnóstico da infecção por HPV. Revista de Ciências Médicas e Biológicas. http://dx.doi.org/10.9771/cmbio.v18i3.34480

Medeiros, F. A., et al. (2010). Acolhimento em uma Unidade Básica de Saúde: a satisfação do usuário em foco. Departamento de Odontologia, Centro de Ciências da Saúde, Universidade Federal do Rio Grande do Norte - UFRN. https://www.scielosp.org/article/rsap/2010.v12n3/402-413/.

Melo, M. C., et al. (2012). O Enfermeiro na Prevenção do Câncer do Colo do Útero: o Cotidiano da Atenção Primária. Revista Brasileira de Cancerologia. https://rbc.inca.gov.br/site/arquivos/n_58/v03/pdf/08_artigo_enfermeiro_prevencao_cancer_colo_utero_cotidiano_atencao_pr ima ria.pdf

Mendes, K. D. S., Silveira, R. C. C. P., Galvão, C. M. (2008). Revisão integrativa: método de pesquisa para a incorporação de evidências na saúde e na enfermagem. Texto Contexto Enferm, 17(4): 758-64.

Nascimento, L. C., et al. (2012). Conhecimento cotidiano de mulheres sobre a prevenção do câncer de colo do útero. Rev. enferm. UERJ. https://www.epublicacoes.uerj.br/index.php/enfermagemuerj/article/view/4813/3537

Nascimento, M. I., et al. (2020). Mortalidade prematura por câncer de colo uterino: estudo de séries temporais interrompidas. Rev. Saúde Pública 54. https://doi.org/10.11606/s1518-8787.2020054002528

Oliveira, J. T., et al. (2017). Intervenções de enfermagem na prevenção do câncer cérvico-uterino: perspectivas das clientes. Rev enferm UERJ. https://docs.bvsalud.org/biblioref/2018/10/915884/26242-103129-1-pb.pdf

Omasi, E., et al. (2015). Estrutura e processo de trabalho na prevenção do câncer de colo de útero na Atenção Básica à Saúde no Brasil: Programa de Melhoria do Acesso e da Qualidade - PMAQ-Recife. http://www.scielo.br/scielo.php?script=sci_abstract\&pid=S1519-38292015000200171\&lng=pt\&nrm=iss

Ramos de Souza, K, et al. (2015). Educação popular como instrumento participativo para a prevenção do câncer ginecológico: Percepção de mulheres. http://dx.doi.org/10.15649/cuidarte.v6i1.129.

Santiago, T. R., et al. (2014). Conhecimento e prática das mulheres atendidas na unidade de saúde da família sobre o Papanicolaou. Rev. enferm. UERJ. https://pesquisa.bvsalud.org/portal/resource/pt/bde-27923

Santos, M. S. D., et al. (2011). Saberes e práticas de mulheres idosas na prevenção do câncer cérvico-uterino. Rev. bras. Enferm. http://dx.doi.org/10.1590/S0034-71672011000300009.

Santos, N. F., et al. (2019). Acesso ao exame citológico do colo do útero em região de saúde: mulheres invisíveis e corpos vulneráveis. Cad. Saúde Pública 35 (10). https://doi.org/10.1590/0102-311X00234618

Silva, M. M., et al. (2012). Atenção básica em saúde: prevenção do câncer de colo do útero na consulta de enfermagem. Revista Brasileira de Cancerologia. https://rbc.inca.gov.br/site/arquivos/n_58/v03/pdf/08_artigo_enfermeiro_prevencao_cancer_colo_utero_cotidiano_atencao_primaria.pdf

Silva, R. C. G., et al. (2018). Perfil de mulheres com câncer de colo do útero atendidas para tratamento em centro de oncologia. Rev. Bras. Saude Mater. Infant. 18(4). https://doi.org/10.1590/1806-93042018000400002

Soares, M. C., et al. (2011). Câncer de colo uterino: atenção integral à mulher nos serviços de saúde. Rev. Gaúcha Enferm. http://dx.doi.org/10.1590/S198314472011000300010. https://www.scielo.br/scielo.php?script=sci_arttext\&pid=S1983-14472011000300010 \&lang=pt\#: :text=Comprehensive\%2 0health\% 20care. \&text=O\%20c\%C3\%A2ncer\%20de\%20col o\%20uterino,vida\%20familiar\%2C\%20profissional\%20e\%20social.

Souza, D. M. N., et al. (2018). Desenvolvimento de protocolo clínico para detecção de lesões precursoras do câncer de colo uterino. Rev. Latino-Am. Enfermagem. 26. https://doi.org/10.1590/1518-8345.2340.2999

Souza, M., Mandu, E. N. T., Elias, A. N. (2013). Percepções de enfermeiros sobre seu trabalho na estratégia saúde da família. enferm. 22(3). 
Research, Society and Development, v. 10, n. 4, e1210413728, 2021

(CC BY 4.0) | ISSN 2525-3409 | DOI: http://dx.doi.org/10.33448/rsd-v10i4.13728

Souza, S. A. N., Souto, G. R., Santos, W. L. D. (2020). Assistência da enfermagem relacionada ao câncer uterino. Revista JRG de Estudos Acadêmicos. 3(6) (2020). https://doi.org/10.5281/zenodo.4292286

Vale, D. B., et al. (2010). Avaliação do rastreamento do câncer do colo do útero na Estratégia Saúde da Família no Município de Amparo, São Paulo, Brasil. Cad. Saúde Pública. https://doi.org/10.1590/S0102-311X2010000200017.

Vasconcelos, C. T. M, et al. (2011). Conhecimento, atitude e prática relacionada ao exame colpocitológico entre usuárias de uma unidade básica de saúde. Rev. Latino-Am. Enfermagem. https://doi.org/10.1590/S0104-11692011000100014.

Viana, M. P., et al. (2013). Formação do enfermeiro para a prevenção do câncer de colo uterino. Rev. enferm. UERJ, https://www.epublicacoes.uerj.br/index.php/enfermagemuerj/article/view/10038/7824 